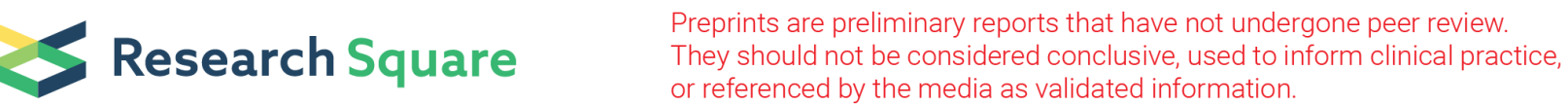

\section{An App Developed for Predicting Nurse Intention to Quit the Job (NIQJ) Using the Artificial Neural Networks(ANN) in Microsoft Excel: Population- Based Questionnaire Study}

\section{Tung-Hui Jen}

Chi Mei Medical Center Chiali Branch: Chi Mei Hospital Chiali

\section{Tsair-Wei Chien}

Chi Mei Medical Center https://orcid.org/0000-0003-1329-0679

\section{Yu-Tsen Yeh}

St. George School: Saint George School

Huan-Fang Lee ( $\square$ eamonn0330@gmail.com )

National Cheng Kung University https://orcid.org/0000-0002-1276-4409

\section{Research article}

Keywords: nurse intention to quit the job, artificial neural network, convolutional neural network, nurse, Microsoft Excel, receiver operating characteristic curve

Posted Date: January 26th, 2021

DOl: https://doi.org/10.21203/rs.3.rs-152558/v1

License: (c) (1) This work is licensed under a Creative Commons Attribution 4.0 International License. Read Full License 


\section{Abstract}

Background: Studies in the past have identified factors related to the nursing staff's intention to leave the unit, institution, and profession. However, none has successfully predicted the nurse's intention to quit the job (NIQJ). Whether NIQJ can be predicted be predicted is an interesting topic in healthcare management. A model to predict the NIQJ for novice nurses in hospitals should be investigated and developed in this mobile computer age.

Objective: The aim of this study is to build a model to develop an app for automatic prediction and classification of NIQJ using a smaller number of items to help assess NIQJ and take necessary actions before nurses quit the job.

Methods: We recruited 1104 novice nurses working in six medical centers in Taiwan to complete 100-item questionnaires related to NIQJ in October 2018. The k-mean was used to divide nurses into two classes (i.e., NIQJ and Non- NIQJ) based on five- NIQJ items regarding leave intention. Feature variables were chosen from 100 relevant items. Two models, including artificial neural network (ANN) and convolutional neural network $(\mathrm{CNN})$, were compared across four scenarios made up by two training sets $(\mathrm{n}=1104$ and $n=804 B)$ and their corresponding testing ( $n=300 a)$ sets to verify the model accuracy (e.g., sensitivity, specificity, area under the receiver operating characteristic curve, AUC) and stability and generalization (e.g., using the training set to predict the testing set). An app predicting NIQJ was then developed involving the model's estimated parameters as a website assessment.

Results: We observed that (1) 24 feature variables extracted from this study in ANN model yielded a higher AUC of 0.82 (95\% Cl 0.80-0.84) based on the total 1104 cases, (2) the ANN performed better than CNN on both accuracy, stability and generalization, and (3) an ready and available app for predicting NIQJ was successfully developed in this study.

Conclusions: The 24-item ANN model with the 53 parameters estimated by the ANN for improving the accuracy of NIQJ has been developed with the use of Excel (Microsoft Corp). The app would help team leader and HR department to pick up nurse's NIQJ before actions are taken, allowing them to make plans accordingly.

\section{Highlights}

- We performed ANN on Microsoft Excel, which is rare in the literature.

- An app was built to display results using a visual dashboard on Google Maps. The animationfeatured dashboard was incorporated with the ANN model and allow an easy understanding of the classification results with visual representations.

- The category probability curves were uniquely derived from the Rasch rating scale model and launched to the ANN prediction model to display the binary classification, using probability to interpret the prediction results. 


\section{Background}

The World Health Organization (WHO)[1] reported that although there is a gradual increase in the number of medical professionals around the world, the figures are far from meeting the rising demands[2] for health care manpower, nursing staff in particular. More than half of nurses with the National Health Service(NHS) have considered quitting the work pressures continue to ratchet up[3,4].

Lack of nursing staff may lead to increased medical incidence because of negligence, involving infection, falls, medication errors, tube dislodgement, pressure sores, and, most seriously, death $[5,6]$. Nurses overloaded with a high number of patients may experience increased work stress, which has been identified as a key factor in increasing nurse turnover [7]. The excessive workload on nursing staff may prolong patient hospitalization, increase patient morbidity and mortality, and increase the incidence of adverse events [8]. Therefore, it is just as important for hospital management to predict Nurses Intention

to Quit the Job (NIQJ) as it is to investigate the reason behind the insufficient nursing workforce [9].

Numerous studies [10-13] have identified factors related to a nurse's intention to leave the unit, institution, and profession. However, there has not been any success in predicting NIQJ using the technique of artificial neural network (ANN) in literatures. The advance computer technique has enabled us to overcome the failure in prediction. Early identification of NIQJ may prompt the involvement of supporting service and subsequently alter the outcome, whether it is a longer career for nurses or a buffer period for the management team to make arrangements.

\section{Artificial Neural Network}

The ANN is a component of artificial intelligence that is meant to simulate a functioning human brain[14]. ANN is the foundation of artificial intelligence (Al) and solves problems that would otherwise be impossible or very difficult by human statistical standards[15]. It was worthy incorporating ANN, the famous deep learning method, to see if it can improve the prediction accuracy on NIQJ classification without having to directly ask the question of quitting[16].

\section{Online Classification Using Smartphones is Required}

As with the advancements in web-based technologies, mobile health communication is rapidly improving[17]. There was no smartphone app designed to classify NIQJ yet. Once the ANN algorithm learned and estimated the NIQJ model's parameter, the classification system can incorporate an early warning response for HR management to react only to those directly relevant to quitting as well as to plan ahead accordingly[16].

\section{Study Aims}

The aims of our study are to (1) estimate the model's parameters using ANN based on nurse's responses to questionnaires on both NIQJ and Non- NIQJ and (2) design smartphones based app for assessment on NIQJ. 


\section{Methods}

\section{Study Sample and Demographic Data}

If the confidence level and intervals are set at 0.05 and $\pm 5 \%$ and applies to the population of 300 novice nurses (about $30 \%$ of nurses in hospitals are NO and N1 in the nursing hierarchy [18]) in a hospital, 169 participants are required to fulfill adequate sample size $[19,20]$. We estimated the rate of refusal to respond to be around $40 \%$. Therefore, the minimum number of participants for this study will be 282 $(169 /[1-0.4])$.

In October 2018, we delivered 300 copies each to six medical centers in Taiwan, inviting novice nurses(i.e., nurse hierarchy at N0 and N1 only) to complete the 100-item questionnaires(Figure 1 and Additional File1) related to NIQJ[10-13]. A total of 1,104 nurses participated, with a return rate of $61.3 \%$ (Additional File2).

This study was approved and monitored by the NCKU Hospital institutional review board (06476734). All hospital and participants' identifiers were stripped.

\section{Featured Variables}

Featured variables were extracted from these 100-item questionnaires using logistic regression with Type error set at 0.05, where the dependent variable(NIQJ as 1 and Non- NIQJ as 0) was determined by k-mean clustering method[21] on the summation scores of NIQJ(Figure 1).

\section{Four scenarios and two models}

Model accuracy(e.g.,>0.7) and stability(or, say, generatlization) (e.g. discrepancy between training and testing sets) were focused on out of various facets like model feasibility, efficacy, and efficiency. Firstly, the 1,104 participants were split into training and testing sets in a proportion of $70 \%$ to $30 \%$, where the former was used to predict the latter. Four scenarios consisting two training and two testing sets derived from such grouping ratio: Total cases $(n=1104)$ as a training set, its corresponding testing set $(n=300)$, another training sets using $70 \%$ of participants $(n=804)$, and its corresponding testing set $(n=300)$. The higher and lower summation scores of NIQJ were used in the training sets, while the middle summation scores of NIQJ were used in the testing sets. Secondly, the accuracy (e.g., sensitivity, specificity, area under the receiver operating characteristic curve, AUC) and stability(or, generalization) ( (e.g., using the training set to predict the testing set) were verified. The data is shown in Multimedia Appendix 2.

The ANN and convolutional neural network (CNN) were analyzed with the four scenarios mentioned above. CNN has traditionally been performed on Microsoft (MS) Excel[18, 20,22] while ANN has not been paired along with MS Excel in the past. As demonstrated in figure 2 below, the ANN process involves data input in layer 1 where the data joined with two types of parameters and run through the sigmoid function algorithms in layers 2 and 3 . Finally, as shown on the right side and bottom of figure 2, the prediction 
model was deemed complete when the total residuals were minimized through the MS Excel function of sumxmy 2 and solver add-in.

\section{Tasks for Performing ANN and CNN}

\section{Task 1: Comparison of Accuracies on Two Models and Stability across Four Scenarios}

The accuracy was determined by observing the higher indicators of sensitivity, specificity, precision, F1 score, accuracy, and AUC in both models. The definitions are listed below:

True positive (TP)=the number of predicted NIQJ to the true NIQJ, (1)

True negative $(T N)=$ the number of predicted Non-NIQJ to the true Non-NIQJ, (2)

False-positive (FP) $=$ the number of Non-NIQJ minuses TN, (3)

False-negative $(\mathrm{FN})=$ the number of NIQJ minuses TP, (4)

Sensitivity $=$ true positive rate $(T P R)=T P(T P+F N),(5)$

Specificity $=$ true negative rate $(T N R)=T N(T N+F P),(6)$

Precision= positive predictive value $(\mathrm{PPV})=\mathrm{TP}(\mathrm{TP}+\mathrm{FP}),(7)$

F1 score=2(8)

$A C C=$ accuracy $=(T P+T N) N,(9)$

$\mathrm{N}=\mathrm{TP}+\mathrm{TN}+\mathrm{FP}+\mathrm{FN},(10)$

AUC $=(1-$ Specificity)SensitivitySpecificity, (11)

SE for $A U C==,(12)$

$95 \% \mathrm{Cl}=\mathrm{AUC} \pm 1.96,(13)$

The stability was determined by observing the AUC changes in both training and testing sets. The fewer AUC changes in a prediction model imply better stability. Comparisons of AUCs across four scenarios were made in both ANN and CNN models.

\section{Task 2: App Developed for Predicting NIQJ}

A self-assessment app using participant mobile phones was designed to predict NIQJ using the ANN(or $\mathrm{CNN}$ ) algorithm with the model parameters. The result is shown as classification then appears on smartphones. The visual representation with binary (NIQJ and Non-NIQJ) category probabilities is shown on a dashboard using Google Maps. 


\section{Statistical Tools and Data Analysis}

IBM SPSS Statistics 22.0 for Windows (SPSS Inc) and MedCalc 9.5.0.0 for Windows (MedCalc Software) were used to perform descriptive statistics, frequency distributions among groups, logistic regression analyses, and the computation of model prediction indicators mentioned in Eqs 1 to 13. The significant level of type I error was set at 0.05. ANN and CNN were performed on MS Excel (Microsoft Corp).

A visual representation of the classification was plotted using two curves based on the Rasch model [23]. The study flowchart and the ANN modeling process are shown in Figure 3 and Multimedia Appendix 3 , respectively.

\section{Results}

\section{Demographic Data of Participants}

The demographic data of the novice nurses (i.e., nurse hierarchy at N0 and N1) are shown in Table 1. Two of the medical centers, $B(n=134)$ and $F(n=135)$, had few participants than the minimal satisfactory sample size of 169. Most participants were aged below 30(1010/1104=91.5\%) and worked for privatelyrun hospitals $(667 / 1104=60.4 \%)$. Normal distributions were seen in self-assessment of health status and workload. $16.9 \%$ identify themselves as being religious. The number of positive NIQJ(=571) is slightly higher than those with Non-NIQJ(=533).

\section{Featured variables extracted from data}

24 featured variables out of the original 100 items (Figure 1) were identified as statistically significant $(p<0.05)$ using the multiply logistic regression, with a threshold of the dependent variable(NIQJ) composed scores at $<12$ (Non-NIQJ) using k-mean clustering method .

\section{Accuracy and Stability as well as generalization in comparison of models}

When comparing the two models with full data set of 1104 cases, the ANN model scored higher than the CNN model across all six indicators of sensitivity, specificity, precision, F1 score, accuracy, and AUC, suggesting that the ANN model had a higher accuracy.

Thee ANN model also performs better in terms of model stability when comparing the testing results with ACUs (e.g., $0.68>0.59$ and $0.78>0.71$ in Table 2 and the lower AUC of testing 300 in Figure 4). It is worth mentioning that the group consisting $70 \%$ of sample $(n=804)$ has the highest AUC when comparing to other scenarios due to the higher discrimination power caused by selecting, the lower and higher summation scores in NIQJ, as mentioned in the method section.

\section{App Predicting NIQJ for a Web-Based Assessment}

The interface of the app targeting novice nurses in order to predict NIQJ was shown on the left-hand side of Figure 5. Readers are invited to click on the links[24,25] and interact with the NIQJ app, see Multimedia 
Appendix 4. It is worth noting that all 53 model parameters are embedded in the 24-item ANN model. Once responses are submitted, it generates a result as a classification of either possible NIQJ and NonNIQJ without having to directly ask the question regarding quitting.

An example is shown on the right-hand side of Figure 5, from which we can see that the participant scored a moderate probability (0.83) of non-NIQJ, which is the curve starting from the top left to the bottom right corner. The sum of probabilities for NIQJ and Non-NIQJ is 1.0. The odds can be calculated with the formula $(p /[1-p]=0.17 / 0.83=0.20)$, suggesting that this novice nurse has an extremely low probability or tendency to quit.

\section{Discussion}

\section{Principal Findings}

We observed that (1) 24 featured variables identified in this study yielded a higher AUC of $0.82(95 \% \mathrm{Cl}$ 0.80-0.84) with the ANN model based on the 1104 cases, (2) the ANN performs better in both accuracy and stability than the CNN, and (3) a possible app for predicting NIQJ was successfully developed and demonstrated in this study.

Previous studies[10-13] merely identified the factors related to the nursing workers' intention to leave the unit, health institution, and profession. Over 476 articles came up with the keyword (nurse intention to leave)[26] on PubMed Central as of October 11, 2020. There has not been a predictive model built for analyzing NIQJ. Although authors developed a Support Vector Machine for predicting nurses' intention to quit using working motivation, job satisfaction, and stress levels as predictors[16], none has demonstrated an online predictive model as we did in this study.

More than half of nurses with the National Health Service(NHS) have considered quitting work pressures continue to ratchet up [3,4]. Although the intention to leave dose not always lead to action(or behavior) [27], predicting nurses' intention to quit is an essential and necessary approach to set up an early warning mechanism in the scope of human resource management[16], considering the constant shortage of nursing staff and the current increasing demands1, 2]. In this study, we verified that the ANN could improve the prediction accuracy on NIQJ classification, which is novel and innovative, where predictions are made without having to ask the direct question regarding quitting.[16].

\section{Implications and Future Work}

The ANN performed better than the CNN in both accuracy and stability. In this study, the sensitivity and specificity have been improved. We have not seen others using the ANN approach to predict NIQJ in the literature, which is a breakthrough in this study. We have also not noticed any article incorporating accuracy and stability as well as a generalization to verify model feasibility, efficacy, and efficiency, but many authors have used the split scheme of $70: 30$ ratio invalidating their predictive models $[18.20,28]$ 
Over 2,062 articles have been found searching the keyword (artificial neural network) [Title] on PubMed Central on October 10, 2020. None of them used Microsoft Excel to perform the ANN. The interpretations for the ANN concept and process, as well as the parameter estimations, are shown in Figure 2, Multimedia Appendix 3, and in the app [24,25]. Readers can adjust the parameters in the ANN model on their own and examine the difference in results.

As the quality control process emphasized the principle to consider more with the vital few and less with the trivial numerous[20], we suggest adapting with the matching personal response scheme for correct classifications in the model (MPRSA) and further increase the accuracy toward 100\%[20] in the ANN model. Because the reason being that the same response string will be matched by the MPRSA and lead to a correct classification if the responses are identical to that of the original dataset. We recommend searching individual responses in the dataset first. If found, assign the correct classification to this respondent. Otherwise, the classification will be determined by the NIQJ ANN model; see Multimedia Appendix 5.

Furthermore, the curves of category probabilities based on the Rasch model [23] are shown in Figure 5. The binary categories (e.g., success, and failure on an assessment in the psychometric field) have been applied in health-related outcomes [29-33]. However, we are the first to provide the NIQJ animation-type dashboard on Google Maps, as shown in Figure 5.

\section{Strengths of this study}

ANN was performed on Microsoft Excel, which is rare in the literature. An app was designed to display classification results using the category probability theory in the Rasch model. The animation-featured dashboard was incorporated with the ANN model and allow an easy understanding of the classification with visual representations.

There are different types of algorithms for classification in Machine Learning[34,35], such as Logistic Regression, Support Vector Machines(SVM)[36], Naïve Bayes, Random Forest Classification, ANN, CNN[18,20,22], and k-nearest neighbors algorithm (KNN)[37]. ANN was shown superior with $93.2 \%$ classification accuracy in the previous study[36], similar to our results, although only two models(CNN and ANN) were compared.

\section{Limitations and Suggestions}

Our study has some limitations. First, although the psychometric properties of the 24-item NIQJ assessment have been validated, there is no evidence to support that it is suitable for novice nurses in other regions. We recommend additional studies using the same approach with the ANN or other models to estimate the parameters and explore the difference and similarity to this study.

Second, we have not discussed possible further improvement in predictive accuracy. For instance, whether other featured variables (e.g., variables are not included in Figure 1) applied to the ANN model 
can increase the accuracy rate is worth discussion. It would be useful in the future to look for other variables that can improve the power of the model prediction.

Third, the study was carried out on the ANN model whether other predictive models have higher accuracy and stability than the ANN has yet to be investigated.

Finally, the study sample was taken from novice nurses in Taiwan. The model parameters estimated for the NIQJ are only suitable for the Chinese (particularly Taiwanese) health care settings. Generalizing these NIQJ assessment findings (e.g., the model parameters) might be difficult and constrained because the sample only took into consideration novice nurses working for inpatients. Additional studies are required to re-examine whether the psychometric properties of the NIQJ assessment are similar for inpatients and other sites..

\section{Conclusion}

We demonstrated in this study: (1) ANN performed on Microsoft Excel, (2) MPRSA is recommended to increase the model's prediction accuracy, (3) an online app made to display results using a visual dashboard on Google Maps, and (4) the category probability curves based on Rasch model combined with the ANN prediction model. The novelty of the app with our ANN algorithm improves the predictive accuracy of the NIQJ. The integration of this app would hopefully help the team leader and HR department to pick up nurse's NIQJ before actions are taken, allowing them to make plans accordingly.

\section{Abbreviations}

Al: artificial intelligence

ANN: artificial neural network

AUC: Area under ROC curve

CNN: convolutional neural network

KNN: k-nearest neighbors algorithm

MPRSA: matching personal response to adapt for the correct classification

NIQJ: nurse intention to quit the job

SD:standard deviation

SVM: Support Vector Machines

ROC:Results: a receiver-operating characteristic 
WHO: World Health Organization

\section{Declarations}

\section{Ethics approval and consent to participate}

This study was approved and monitored by the NCKU Hospital institutional review board (06476734). All hospital and participants' identifiers were stripped.

\section{Consent to publish}

Not applicable.

\section{Availability of data and materials}

All data used in this study are available in Additional File files.

\section{Competing interests}

The authors declare that they have no competing interests.

\section{Funding}

There are no sources of funding to be declared.

\section{Authors' Contributions}

TH conceived and designed the study. YT performed the statistical analyses and was in charge of recruiting study participants. TWC helped design the study, collected information and interpreted data. HFL monitored the research. All authors read and approved the final article.

\section{Acknowledgments}

We thank Enago (www.enago.tw) for the English language review of this manuscript. All authors declare no conflicts of interest.

\section{References}

1. World Health Organization. Global strategy on human resources for health: Workforce 2030. Switzerland, Geneva: World Health Organization. 2016. 2020/10.10 available at https://www.who.int/hrh/resources/globstrathrh-2030/en/

2. Babalola O. Consumers and their demand for healthcare. Medical economics 2017;3(1):1-6.

3. Dean E. Half of nurses say they want to quit the profession. Nurs Manag (Harrow). 2017 Mar 30;24(1):6. doi: 10.7748/nm.24.1.6.s2. PMID: 28357942. 
https://pubmed.ncbi.nlm.nih.gov/28357942/

4. Dean E. Half of nurses considering leaving NHS, survey finds. Nurs Stand. 2017 Mar 22;31(30):7-8. doi: 10.7748/ns.31.30.7.s3. PMID: 28327015.

5. Dunton N, Gajewski B, Taunton RL, Moore J. Nurse staffing and patient falls on acute care hospital units. Nursing Outlook 2004; 52(1): 53Y58. doi:10.1016/j.outlook.2003.11.006

6. Rafferty AM, Clarke SP, Coles J, Ball J, James P, McKee M, Aiken LH. Outcomes of variation in hospital nurse staffing in English hospitals: Cross-sectional analysis of survey data and discharge records. International Journal of Nursing Studies 2007; 44(2): $175 Y 182$.

7. Huang TL,Wu JH, Chien TW. Using determination coefficient and Rasch model to innovatively inspect nurse-patient ratios among Taiwan hospitals. The International Journal of Organizational Innovation 2019;12(2):192-204.

8. Aiken LH, Clarke SP, Sloane DM, Sochalski J, Silber JH. Hospital nurse staffing and patient mortality, nurse burnout, and job dissatisfaction. The Journal of the American Medical Association 2002; 288(16): 1987Y1993. doi:10.1001/jama.288.16.1987

9. Lin CF, Huang HY, Lu MS.The development of nursing workforce allocation standards for acute care general wards in Taiwan. The Journal of Nursing Research 2003; 21(4): 298-306.

10. Bordignon M, Monteiro MI. Predictors of nursing workers' intention to leave the work unit, health institution and profession. Rev Lat Am Enfermagem. 2019 Dec 5;27:e3219. doi: 10.1590/15188345.3280.3219. PMID: 31826161; PMCID:

11. Nantsupawat A, Kunaviktikul W, Nantsupawat R, Wichaikhum OA, Thienthong H, Poghosyan L. Effects of nurse work environment on job dissatisfaction, burnout, intention to leave. Int Nurs Rev. 2017 Mar;64(1):91-98. doi: 10.1111/inr.12342. Epub 2016 Nov 24. PMID: 27882573.

12. Moloney W, Boxall P, Parsons M, Cheung G. Factors predicting Registered Nurses' intentions to leave their organization and profession: A job demands-resources framework. J Adv Nurs. 2018 Apr;74(4):864-875. doi: 10.1111/jan.13497. Epub 2017 Dec 5. PMID: 29117451.

13. de Oliveira DR, Griep RH, Portela LF, Rotenberg L. Intention to leave profession, psychosocial environment and self-rated health among registered nurses from large hospitals in Brazil: a crosssectional study. BMC Health Serv Res. 2017 Jan 10;17(1):21. doi: 10.1186/s12913-016-1949-6. PMID: 28068999; PMCID: PMC5223488.

14. Falissard L, Morgand C, Roussel S, Imbaud C, Ghosn W, Bounebache K, Rey G. A Deep Artificial Neural Network-Based Model for Prediction of Underlying Cause of Death From Death Certificates: Algorithm Development and Validation. JMIR Med Inform. 2020 Apr 28;8(4):e17125. doi: 10.2196/17125. PMID: 32343252; PMCID: PMC7218605.

15. Frenkenfield J. Artificial neural network (ANN).2020/10/10 available at https://www.investopedia.com/terms/a/artificial-neural-networks-ann.asp

16. Tzeng HM, Hsieh JG, Lin YL. Predicting nurses' intention to quit with a support vector machine: a new approach to set up an early warning mechanism in human resource management. Comput 
Inform Nurs. 2004 Jul-Aug;22(4):232-42. doi: 10.1097/00024665-200407000-00012. PMID: 15494654.

17. Mitchell SJ, Godoy L, Shabazz K, Horn IB. Internet and mobile technology use among urban African American parents: survey study of a clinical population. J Med Internet Res. 2014 Jan 13;16(1):e9. doi: 10.2196/jmir.2673.

18. Ma SC, Chou W, Chien TW, et al. An App for Detecting Bullying of Nurses Using Convolutional Neural Networks and Web-Based Computerized Adaptive Testing: Development and Usability Study. JMIR Mhealth Uhealth. 2020;8(5):e16747. Published 2020 May 20. doi:10.2196/16747

19. Survey System.[2020-01-14]. Sample Size Calculator https://www.surveysystem.com/sscalc.htm.

20. Lee YL, Chou W, Chien TW, Chou PH, Yeh YT, Lee HF. An App Developed for Detecting Nurse Burnouts Using the Convolutional Neural Networks in Microsoft Excel: Population-Based Questionnaire Study. JMIR Med Inform. 2020 May 7;8(5):e16528. doi: 10.2196/16528. PMID: 32379050; PMCID: PMC7243132.

21. Chen CW, Luo J, Parker KJ.Image segmentation via adaptive K-mean clustering and knowledgebased morphological operations with biomedical applications.IEEE Trans Image Process. 1998;7(12):1673-83.

22. Yan YH, Chien TW, Yeh YT, Chou W, Hsing SC. An App for Classifying Personal Mental Illness at Workplace Using Fit Statistics and Convolutional Neural Networks: Survey-Based Quantitative Study. JMIR Mhealth Uhealth. 2020;8(7):e17857. Published 2020 Jul 31. doi:10.2196/17857

23. Rasch G. Probabilistic Models for Some Intelligence and Attainment Tests.Chicago, US: University of Chicago Press; 1980.

24. Chien TW. NIQJ assessment. 2020/10/10 available at http://www.healthup.org.tw/irs/annnursequit2020.asp

25. Chien TW. NIQJ assessment and questionnaire. 2020/10/10 available at http://www.healthup.org.tw/irs/irsin_e.asp?type1=95

26. Chien TW. Search for nurse intention to leave and quit the job. 2020/10/11 available at https://pubmed.ncbi.nlm.nih.gov/? term=nurse+and+\%28\%22intention+to+quit\%22+or+\%22+intention+to+leave\%22\%29\&sort=pubdate

27. Ma SC, Wang HH, Chien TW. Hospital nurses' attitudes, negative perceptions, and negative acts regarding workplace bullying. Ann Gen Psychiatry. 2017 Sep 15;16:33. doi: 10.1186/s12991-0170156-0. PMID: 28936227; PMCID: PMC5603093.

28. Zhang PI, Hsu CC, Kao Y, Chen CJ, Kuo YW, Hsu SL, Liu TL, Lin HJ, Wang JJ, Liu CF, Huang CC. Realtime Al prediction for major adverse cardiac events in emergency department patients with chest pain. Scand J Trauma Resusc Emerg Med. 2020 Sep 11;28(1):93. doi: 10.1186/s13049-020-00786-x. PMID: 32917261; PMCID: PMC7488862.

29. Lee Y, Lin K, Chien T. Application of a multidimensional computerized adaptive test for a Clinical Dementia Rating Scale through computer-aided techniques. Ann Gen Psychiatry. 2019;18:5. doi: 
10.1186/s12991-019-0228-4. https://annals-general-

psychiatry.biomedcentral.com/articles/10.1186/s12991-019-0228-4.

30. Ma S, Wang $\mathrm{H}$, Chien $\mathrm{T}$. A new technique to measure online bullying: online computerized adaptive testing. Ann Gen Psychiatry. 2017;16:26. doi: 10.1186/s12991-017-0149-z. https://annals-generalpsychiatry.biomedcentral.com/articles/10.1186/s12991-017-0149-z.

31. Ma S, Chien T, Wang H, Li Y, Yui M. Applying computerized adaptive testing to the Negative Acts Questionnaire-Revised: Rasch analysis of workplace bullying. J Med Internet Res. 2014 Feb 17;16(2):e50. doi: 10.2196/jmir.2819. https://www.jmir.org/2014/2/e50/

32. Chien T, Lin W. Improving inpatient surveys: web-based computer adaptive testing accessed via mobile phone QR codes. JMIR Med Inform. 2016 Mar 02;4(1):e8. doi: 10.2196/medinform.4313. https://medinform.jmir.org/2016/1/e8/

33. Hulin $\mathrm{Cl}$, Drasgow F, Parsons C. Item Response Theory: Applications to Psychological Measurement. Homewood: Dow \& Jones Irwin; 1983.

34. Mellors BOL, Spear AM, Howle CR, Curtis K, Macildowie S, Dehghani H. Machine learning utilising spectral derivative data improves cellular health classification through hyperspectral infra-red spectroscopy. PLoS One. 2020;15(9):e0238647. Published 2020 Sep 15.

doi:10.1371/journal.pone.0238647

35. Singh VK, Maurya NS, Mani A, Yadav RS. Machine learning method using position-specific mutation based classification outperforms one hot coding for disease severity prediction in haemophilia ' $A$ ' [published online ahead of print, 2020 Sep 11]. Genomics. 2020;S0888-7543(20)30819-3. doi:10.1016/j.ygeno.2020.09.020

36. Al-Yousef A, Samarasinghe S. A Novel Computational Approach for Biomarker Detection for Gene Expression-Based Computer-Aided Diagnostic Systems for Breast Cancer. Methods Mol Biol. 2021;2190:195-208. doi:10.1007/978-1-0716-0826-5_9

37. Zhang PI, Hsu CC, Kao Y, et al. Real-time Al prediction for major adverse cardiac events in emergency department patients with chest pain. Scand J Trauma Resusc Emerg Med. 2020;28(1):93. Published 2020 Sep 11. doi:10.1186/s13049-020-00786-x

\section{Tables}

Table 1. Demographic data of the study sample. 


\begin{tabular}{|c|c|c|c|c|}
\hline \multirow[t]{2}{*}{ Variable } & \multicolumn{2}{|c|}{ Intention to quit the job } & \multirow[t]{2}{*}{$\mathrm{n}$} & \multirow[t]{2}{*}{$\%$} \\
\hline & No & Yes & & \\
\hline A: Eligible sample size & 533 & 571 & 1104 & 100.0 \\
\hline \multicolumn{5}{|l|}{ B: Medical Center } \\
\hline A & 85 & 107 & 192 & 17.4 \\
\hline B & 54 & 80 & 134 & 12.1 \\
\hline C & 93 & 113 & 206 & 18.7 \\
\hline D & 143 & 122 & 265 & 24.0 \\
\hline$E$ & 86 & 86 & 172 & 15.6 \\
\hline $\mathrm{F}$ & 72 & 63 & 135 & 12.2 \\
\hline \multicolumn{5}{|l|}{ C: Hospital type } \\
\hline Public run & 229 & 208 & 437 & 39.6 \\
\hline Private run & 304 & 363 & 667 & 60.4 \\
\hline \multicolumn{5}{|l|}{ D: Religion type } \\
\hline None & 185 & 230 & 415 & 37.6 \\
\hline General folk beliefs & 253 & 249 & 502 & 45.5 \\
\hline Buddhism & 37 & 34 & 71 & 6.4 \\
\hline Christian & 28 & 32 & 60 & 5.4 \\
\hline Catholic & 6 & 4 & 10 & 0.9 \\
\hline I-Kuan Tao & 19 & 16 & 35 & 3.2 \\
\hline Others & 5 & 6 & 11 & 1.0 \\
\hline \multicolumn{5}{|c|}{ E: self-assessing health score(the higher, the healthier) } \\
\hline Low & 144 & 127 & 271 & 24.5 \\
\hline Medium & 270 & 334 & 609 & 55.1 \\
\hline High & 114 & 107 & 223 & 40.2 \\
\hline \multicolumn{5}{|c|}{ F: Self-assessing work-loadings(the higher, the more loadings) } \\
\hline Low & 83 & 75 & 158 & 14.3 \\
\hline Medium & 314 & 382 & 696 & 63.0 \\
\hline High & 136 & 114 & 250 & 22.6 \\
\hline
\end{tabular}




\begin{tabular}{|lllll|}
\hline G:Age & & & & \\
\hline$<30$ years old & 481 & 529 & 1010 & 91.5 \\
$<40$ years old & 45 & 35 & 80 & 7.2 \\
\hline$<50$ years old & 7 & 7 & 14 & 1.3 \\
\hline
\end{tabular}

Note. The cutting point for the summation score of the intention to quit the job set at greater than 11 using the k-mean clustering method

Table 2 Eligible featured variables extracted from the data in this study 


\begin{tabular}{|c|c|c|}
\hline No. & Featured variable & Prob. \\
\hline 1 & Your religion and believing(0-7 automatically transformed into a dummy code) & 0.00 \\
\hline 2 & Your age(years) & 0.03 \\
\hline 3 & Self-assessing your health(1-10 the more, the healthier) & 0.09 \\
\hline 4 & Self-assessing your work loadings(1-10 the more, the heavier) & 0.02 \\
\hline 5 & Self-assessing your work loadings(0-2 the more, the heavier) & 0.03 \\
\hline *6 & $\begin{array}{l}\text { My workplace supports me to participate in on-the-job education and continuing } \\
\text { education courses }(0-6) \text {. }\end{array}$ & 0.00 \\
\hline *7 & $\begin{array}{l}\text { According to the current job market conditions, my salary is appropriate for my job. } \\
(0-6)\end{array}$ & 0.02 \\
\hline *8 & I think my job is guaranteed. $(0-6)$ & 0.06 \\
\hline *9 & I can take care of my work and family needs. (0-6) & 0.03 \\
\hline *10 & When I am at work, I can arrange family care. (0-6) & 0.02 \\
\hline *11 & I am able to participate in the decisions made by my nursing supervisor. $(0-6)$ & 0.02 \\
\hline *12 & I have the autonomy to make decisions about patient care $(0-6)$ & 0.00 \\
\hline a13 & $\begin{array}{l}\text { I feel fatigued when I get up in the morning and have to face another day on the job. } \\
(0-7)\end{array}$ & 0.01 \\
\hline a14 & I feel burned out from my work(0-7, the more, the more burnout) & 0.00 \\
\hline a15 & I feel like I'm at the end of my rope ( $0-7$, the more, the more burnout) & 0.02 \\
\hline b16 & $\begin{array}{l}\text { I can easily understand how my patients feel about things. (0-7, the less, the more } \\
\text { burnout) }\end{array}$ & 0.02 \\
\hline b17 & I feel very energetic(0-7, the less, the more burnout) & 0.01 \\
\hline c18 & I've become more callous toward people since I took this job(0-6) & 0.00 \\
\hline c19 & Overcoming pressure makes me stronger(0-6) & 0.00 \\
\hline c20 & $\begin{array}{l}\text { I would rather take the lead to solve the problem, rather than let others make the } \\
\text { decision }(0-6)\end{array}$ & 0.00 \\
\hline c21 & When changes happen, I can adapt(0-6) & 0.03 \\
\hline c22 & I think I can control my life. (0-6) & 0.01 \\
\hline c23 & $\begin{array}{l}\text { No matter what obstacles are encountered in the journey of life, I will work hard to } \\
\text { achieve my goals }(0-6)\end{array}$ & 0.00 \\
\hline c24 & If necessary, I can do any with unwelcome way to influence others & 0.02 \\
\hline NIQJ & 1. I consider going to quit my current job & \\
\hline
\end{tabular}


2.I investigated the none-nurse job opportunities $\square$

3.I investigated job opportunities in other hospitals

4.I like the current job and would like to stay at the nurse-related job(inverse)

5.I would like to stay at the current nurse professional job

Note.* From totally disagree to totally agree; ${ }^{\text {ab }}$ Frequent annually toward daily; ${ }^{c}$ \&IQJ

From never to always

Table 3 Comparison of statistics in models and scenarios

\begin{tabular}{|c|c|c|c|c|c|c|c|c|}
\hline Model & $\mathrm{n}$ & Sensitivity & Specificity & Precision & $\begin{array}{l}\text { F1 } \\
\text { score }\end{array}$ & Accuracy & AUC & $95 \% \mathrm{Cl}$ \\
\hline \multicolumn{9}{|l|}{ ANN } \\
\hline $\begin{array}{c}{ }^{\mathrm{a}} \text { All } \\
\text { cases }\end{array}$ & 1104 & 0.83 & 0.81 & 0.82 & 0.83 & 0.82 & 0.82 & $\begin{array}{l}0.80- \\
0.84\end{array}$ \\
\hline${ }^{b}$ Learning & 804 & 0.86 & 0.85 & 0.88 & 0.87 & 0.85 & 0.85 & $\begin{array}{l}0.83- \\
0.88\end{array}$ \\
\hline${ }^{\mathrm{a}}$ Testing & 300 & 0.76 & 0.64 & 0.54 & 0.63 & 0.68 & 0.70 & $\begin{array}{l}0.65- \\
0.75\end{array}$ \\
\hline${ }^{\mathrm{b}}$ Testing & 300 & 0.80 & 0.77 & 0.65 & 0.72 & 0.78 & 0.78 & $\begin{array}{l}0.74- \\
0.83\end{array}$ \\
\hline \multicolumn{9}{|l|}{ CNN } \\
\hline $\begin{array}{r}\mathrm{b} \text { All } \\
\text { cases }\end{array}$ & 1104 & 0.76 & 0.77 & 0.78 & 0.77 & 0.76 & 0.76 & $\begin{array}{l}0.74- \\
0.79\end{array}$ \\
\hline aLearning & 804 & 0.84 & 0.69 & 0.78 & 0.81 & 0.77 & 0.77 & $\begin{array}{l}0.74- \\
0.79\end{array}$ \\
\hline${ }^{\mathrm{a}}$ Testing & 300 & 0.79 & 0.48 & 0.45 & 0.58 & 0.59 & 0.64 & $\begin{array}{l}0.58- \\
0.69\end{array}$ \\
\hline $\mathrm{b}$ Testing & 300 & 0.73 & 0.70 & 0.57 & 0.64 & 0.71 & 0.71 & $\begin{array}{l}0.66- \\
0.76\end{array}$ \\
\hline
\end{tabular}

Using parameters in 804 learning cases to validate the results in the testing sample.

b Using parameters in all 1104 cases to validate the results in the testing sample.

\section{Figures}




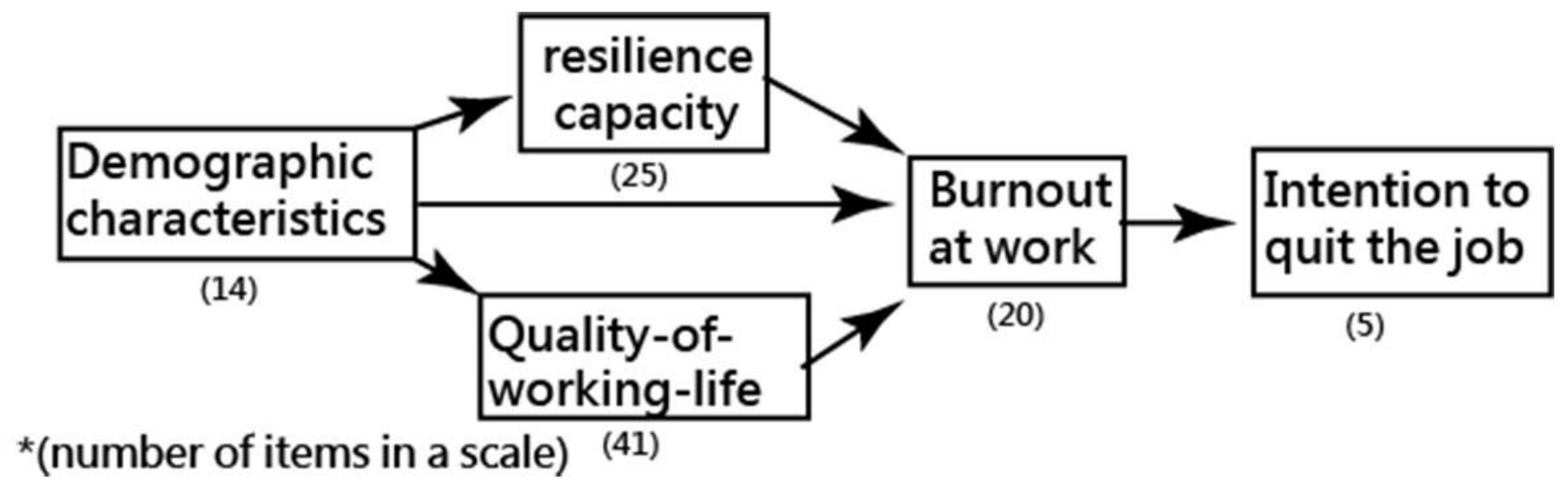

Figure 1

The process of estimating parameters in the ANN model 


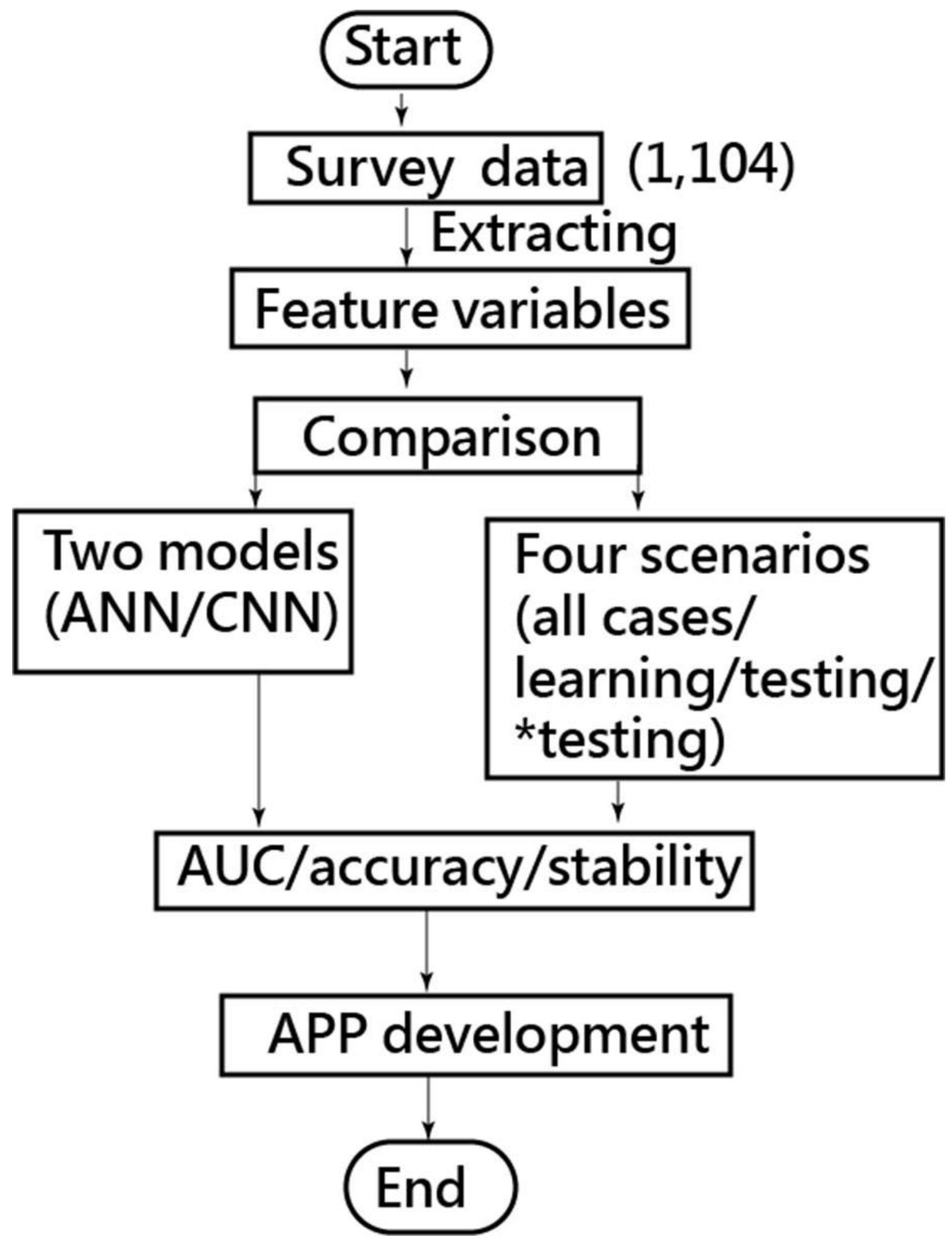

Figure 2

The study flowchart 


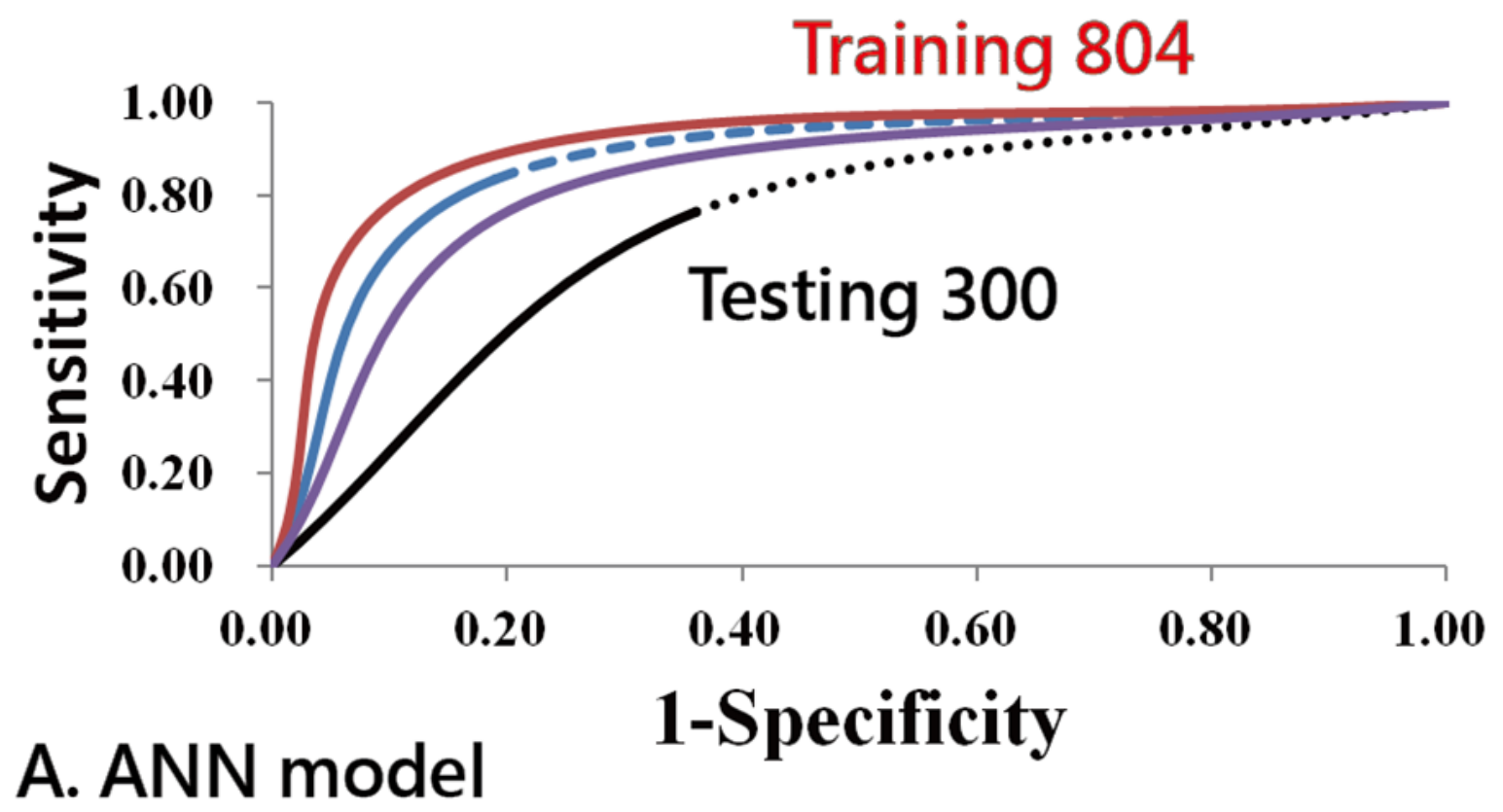

—All 1104 —Training $804-$ Testing 300 —Testing 300

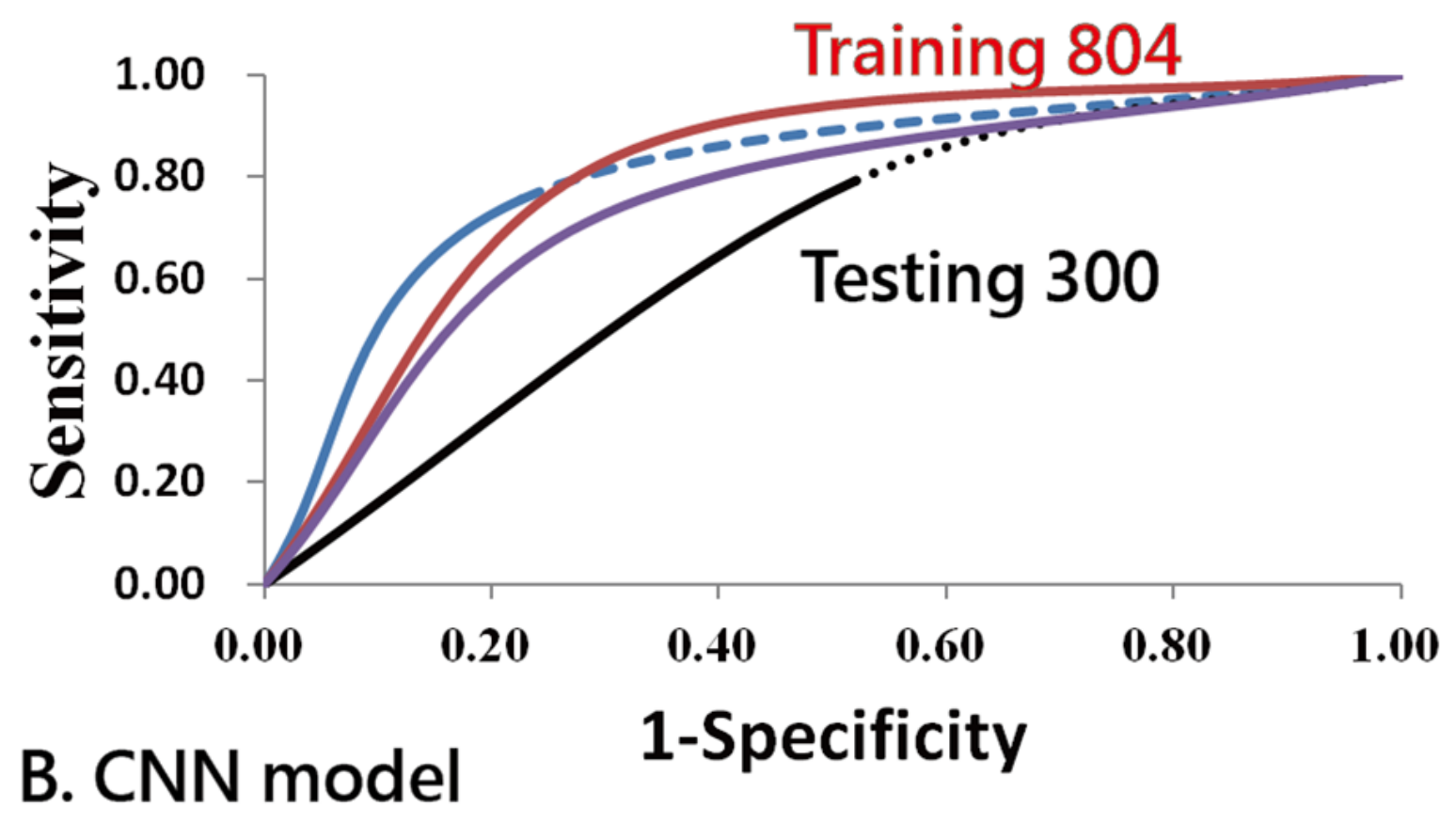

All 1104 — Training 804 — Testing $300 \_$Testing 300

Figure 3

Comparisons of AUC for models and scenarios 
1. Your religion and believing(0-7 automatically transformed into a dummy code) Ans:

\section{Your age(years)}

Ans:

3. Self-assessing your health(1-10 the more, the healthier)

Ans:

4. Self-assessing your work loadings(1-10 the more, the heavier) Ans:

\section{Figure 4}

Feature variables extracted from the 100-NIQJ items for the intention to quit the job

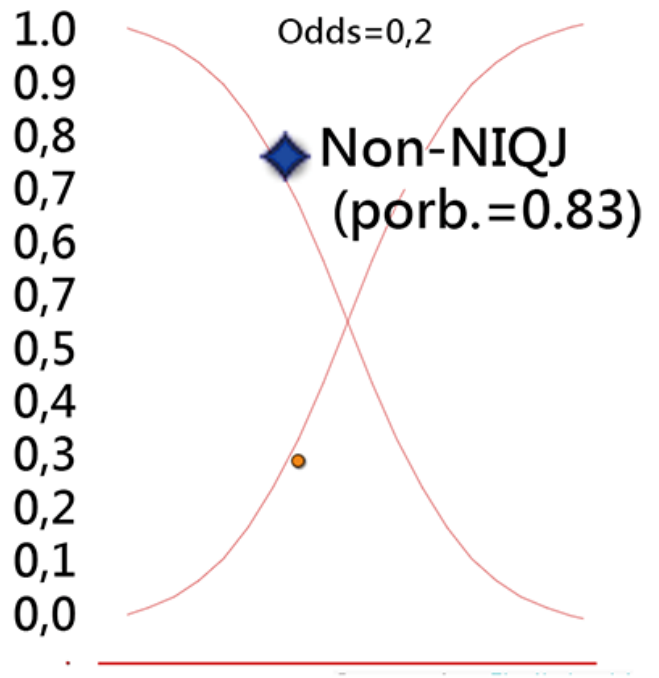



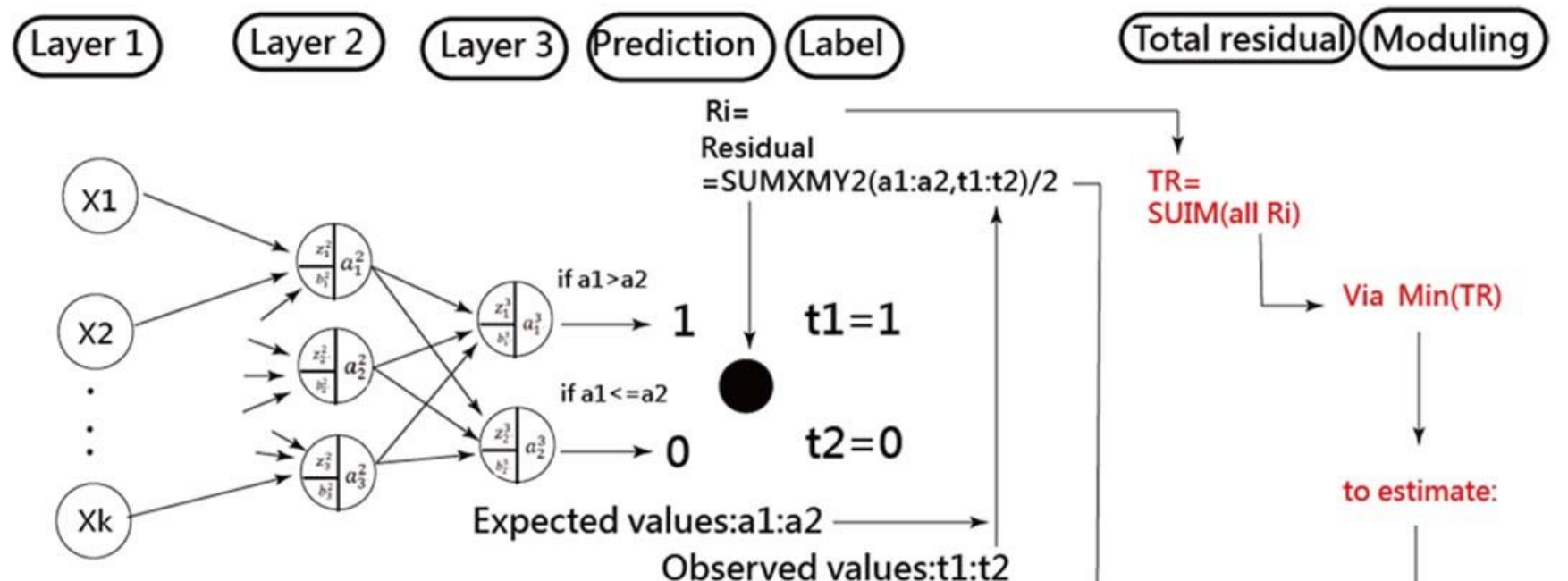

Observed values:t1:t2

A Flnw

Layer $2\left(\begin{array}{c}z_{1}^{2} \\ z_{2}^{2} \\ z_{3}^{2}\end{array}\right)=\left(\begin{array}{ccccc}w_{11}^{2} & w_{12}^{2} & w_{13}^{2} & \ldots & w_{1 k}^{2} \\ w_{21}^{2} & w_{22}^{2} & w_{23}^{2} & \ldots & w_{1 k}^{2} \\ w_{31}^{2} & w_{32}^{2} & w_{33}^{2} & \ldots & w_{1 k}^{2}\end{array}\right)\left(\begin{array}{c}x_{1} \\ x_{2} \\ x_{3} \\ \cdot \\ \cdot \\ \cdot \\ x_{k}\end{array}\right)+\left(\begin{array}{c}b_{1}^{2} \\ b_{2}^{2} \\ b_{3}^{2}\end{array}\right)$

$a_{1}^{2}=a\left(z_{1}^{2}\right), a_{2}^{2}=a\left(z_{2}^{2}\right), a_{3}^{2}=a\left(z_{3}^{2}\right)$, wher $a\left(z_{j}^{j}\right)=$ sigmoid function

$$
=\frac{1}{1+\exp \left(-1 \times z_{i}^{j}\right)} \text {. }
$$

Layer $3 \quad\left(\begin{array}{l}z_{1}^{3} \\ z_{2}^{3}\end{array}\right)=\left(\begin{array}{lll}w_{11}^{3} & w_{12}^{3} & w_{13}^{3} \\ w_{21}^{3} & w_{22}^{3} & w_{23}^{3}\end{array}\right)\left(\begin{array}{l}a_{1}^{2} \\ a_{2}^{2} \\ a_{3}^{2}\end{array}\right)+\left(\begin{array}{l}b_{1}^{3} \\ b_{2}^{3}\end{array}\right)$.

$$
a_{1}^{3}=a\left(z_{1}^{3}\right), a_{2}^{3}=a\left(z_{2}^{3}\right)
$$

Prediction $\quad$
Label
$\mathrm{t} 1=1$
$\mathrm{t} 2=0$
B. Step

Via $\operatorname{Min}(T R)$

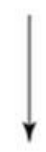

to estimate:

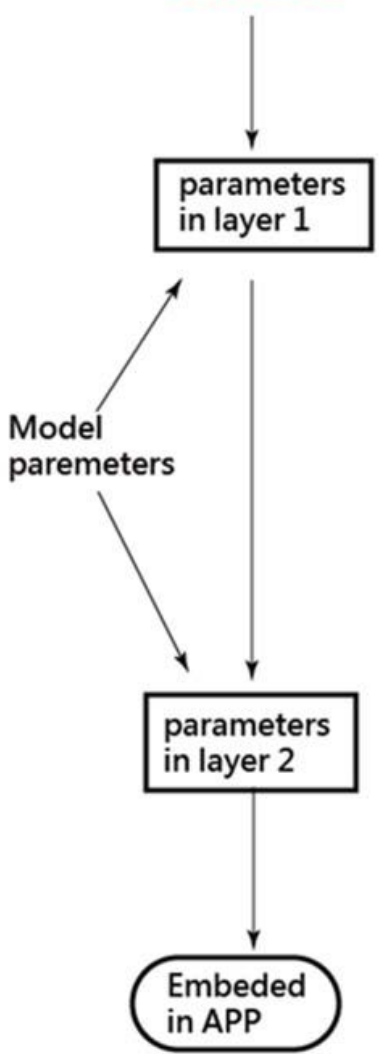

Figure 5

Snapshot of the NIQJ app on a smartphone

\section{Supplementary Files}

This is a list of supplementary files associated with this preprint. Click to download.

- questionnaireenglishversion.docx

- data.txt 
- abstractMP4.txt

- onlineapp.txt

- MPRSAscheme.png

Page 23/23 